\title{
COMPARANDO METODOLOGIAS PARA AVALIAR A COBERTURA DO DOSSEL E A LUMINOSIDADE NO SUB-BOSQUE DE UM REFLORESTAMENTO E UMA FLORESTA MADURA ${ }^{1}$
}

\author{
Márcio Seiji Suganuma ${ }^{2}$, José Marcelo Domingues Torezan ${ }^{3}$, Alba Lúcia Cavalheiro ${ }^{3}$, André Luís Laforga \\ Vanzela $^{3}$ e Táis Benato ${ }^{2}$
}

\begin{abstract}
RESUMO - Os objetivos deste trabalho foram: (1) registrar as vantagens e desvantagens de cinco metodologias utilizadas para avaliar a cobertura do dossel (interpretação de ecounidades, densiômetro esférico, fotografia hemisférica com lente de $8 \mathrm{~mm}$ e fotografia digital com lente de $32 \mathrm{~mm}$ ) e a quantidade de luz que o atravessa (luxímetro e fotografia hemisférica com lente de $8 \mathrm{~mm}$ ); e (2) comparar a estrutura do dossel de um reflorestamento e de um fragmento de Floresta Estacional Semidecídual no norte do Paraná. A classificação em ecounidades é uma metodologia rápida e barata, mas com baixa reprodutibilidade. O densiômetro produz medidas rápidas e confiáveis, e o luxímetro e a fotografia com lente de $32 \mathrm{~mm}$ forneceram dados com pouca precisão, pois são sensíveis a pequenas variações do dossel, e a fotografia com lente de $8 \mathrm{~mm}$ é uma metodologia rápida e de alta precisão, mas apresenta alto custo. Analisando-se a estrutura do dossel, não houve diferenças significativas entre o densiômetro e a fotografia em $8 \mathrm{~mm}$ em nenhum dos dois ambientes; a fotografia em $32 \mathrm{~mm}$ apresentou resultados diferentes, com grande variação nas médias, indicando alta sensibilidade a pequenas alterações no dossel. Na avaliação da quantidade de luz que penetra no sub-bosque, o luxímetro e a lente de $8 \mathrm{~mm}$ foram diferentes. Todas as metodologias apresentaram diferenças entre a floresta madura e o reflorestamento.
\end{abstract}

Palavras-chave: Cobertura do dossel, densiômetro e ecounidades.

\section{COMPARING METHODOLOGIES TO ASSESS CANOPY COVER AND UNDERSTOREY LIGHT ENVIRONMENT OF A REFORESTATION AREA AND A MATURE FOREST}

\begin{abstract}
The objectives of this work were: (1) to investigate the efficiency, advantages and disadvantages of five methodologies to evaluate canopy structure (eco-unit interpretation, spherical densiometer, hemispheric photography with $8 \mathrm{~mm}$ lens and digital photography with $32 \mathrm{~mm}$ lens) and the amount of light crossing it (using light meter and hemispheric photography with $8 \mathrm{~mm}$ lens) and (2) to compare the canopy structure of a reforestation area and a semideciduous forest fragment in northern Paraná. The classification of forest mosaic in eco-units is a fast and cheap methodology, but it lacks repeatability. Densiometer provides fast and reliable measurements, while light meter and $32 \mathrm{~mm}$ lens present data with little precision, being thus sensitive to small variations in the canopy; $8 \mathrm{~mm}$ lens is a fast and high precision methodology but expensive. No significant difference was found in the canopy cover estimates between densiometer and the $8 \mathrm{~mm}$ lens photography in either of the two sites, while $32 \mathrm{~mm}$ lens photography showed significantly different results and great mena variation, due to high sensitivity to the small alterations in the canopy. The luminosity evaluation showed significantly different light meter and $8 \mathrm{~mm}$ lens photography. All the methodologies showed significant differences between the mature forest and the reforestation area.
\end{abstract}

Keywords: Canopy cover, densiometer and eco-units.

\footnotetext{
${ }^{1}$ Recebido em 26.12.2006 e aceito para publicação em 20.02.2008.

${ }^{2}$ Programa de Pós-Graduação em Ciências Biológicas da Universidade Estadual de Londrina(UEL). E-mail:<marviosuganuma@gmail.com>.

${ }^{3}$ Departamento de Biologia Animal e Vegetal da UEL. E-mail: <terezan @uel.br>.
} 


\section{INTRODUÇÃOO}

A luminosidade é um fator determinante nos processos ecológicos e fisiológicos dos vegetais de uma floresta (DENSLOW et al., 1990; TURTON e FREIBURGER, 1997) e pode influenciar, positiva ou negativamente, o desenvolvimento e estabelecimento das plântulas (ALVARENGA et al., 2003; LIMA JR. et al., 2006). Sua variação proporciona grande diversidade de micro-habitats dentro de uma floresta, e a maior causa dessa variação está relacionada com a abertura de clareiras, a complexidade estrutural do dossel e a sua deciduidade (BIANCHINI et al., 2001).

Informações sobre a luminosidade no sub-bosque são essenciais em estudos de florestas tropicais (ENGELBRECHT e HERZ, 2001). O dossel fechado e as clareiras não influenciam somente na incidência da luz e sua composição espectral, mas também a temperatura e umidade relativa do are do solo (CAMPBELL e NORMAN, 1989; RINCON e HUANTE, 1993). Esses parâmetros podem explicar as diferentes formações em um sub-bosque de uma floresta, bem como determinar a dinâmica na regeneração e recrutamento de novas espécies (LAMB et al., 1997). O tipo de estrutura do dossel pode também causar outras conseqüências indiretas, como as variações nos processos de fotossíntese, de transpiração e de alongamento das células vegetais, infecção por patógenos, multiplicação de insetos e competição na comunidade de plantas (CAMPBELL e NORMAN, 1989).

As metodologias utilizadas para mensurar as condições de luminosidade em sub-bosques de florestas são geralmente indiretas, já que medidas diretas como a captação da densidade do fluxo de fótons fotossinteticamente ativos (photosynthetic photon flux density - PPFD) costumam ser trabalhosas e caras (ENGELBRECHT e HERZ, 2001). Assim, este trabalho teve como objetivos: (1) estimar a eficiência e as vantagens e desvantagens de cinco diferentes metodologias, usadas para medir a cobertura de dossel e a quantidade de luz que consegue penetrar no subbosque; e (2) comparar a estrutura do dossel de um reflorestamento com a de uma floresta madura nas diferentes metodologias.

\section{MATERIAL E MÉTODOS}

O estudo foi realizado no Parque Estadual Mata dos Godoy (PEMG), em Londrina, PR (23⒉'S, 51 $\left.{ }^{\circ} 15^{\prime} \mathrm{W}\right)$. O Parque apresenta uma área de 680 ha, com uma vegetação classificada como Floresta Estacional Semidecidual Submontana (SILVEIRA, 2006), composta por uma floresta madura, florestas secundárias e reflorestamentos. As altitudes variam de $500 \mathrm{~m}$ (na parte sul) a $640 \mathrm{~m}$ (na parte norte), com uma precipitação média anual de $1.600 \mathrm{~mm}$ (VICENTE, 2006).

Para a coleta de dados de cobertura do dossel, foram selecionadas duas áreas: (i) a floresta madura (Local-M), na região norte do Parque; e (ii) o reflorestamento (Local-R), na região leste do Parque. O Local-R é um reflorestamento de 40 ha, com cinco espécies nativas de interesse madeireiro (Colubrina glandulosa Perk., Parapiptadenia rigida (Benth.) Brenan, Peltophorum dubium (Spreng.) Taub., Tabebuia impetiginosa (Mart.) Standl., Cordia trichotoma (Vell.) Arrab. Ex Steud.), livre de roçagem e desbaste, plantado em espaçamento de 3 x 3 m em 1990, pelo Instituto Ambiental do Paraná (IAP).

Foram feitos oito transectos, quatro no Local-M e quatro no Local-R, cada um contendo cinco pontos de coleta distantes $40 \mathrm{~m}$ um do outro, com o primeiro localizado a $10 \mathrm{~m}$ da borda. As metodologias utilizadas foram: (1) interpretação de ecounidades (EUs), (2) avaliação da cobertura do dossel com densiômetro esférico, (3) medida de luminosidade na faixa visível do espectro com luxímetro, (4) fotografia digital com lente de $32 \mathrm{~mm}$ e (5) fotografia digital hemisférica com lente de $8 \mathrm{~mm}$ ("olho de peixe").

A interpretação das ecounidades foi feita a partir da identificação de quatro classes de cobertura do dossel (Figura 1): clareira (CL, abertura recente no dossel, provocada pela queda de uma ou mais árvores ou galhos grandes), dossel em regeneração (DR, fase mais avançada da cicatrização da clareira, com espécies arbóreas ultrapassando os $2 \mathrm{~m}$ de altura, associado a crescimento lateral dos ramos das árvores vizinhas e emaranhados de cipós), dossel aberto (DA, com espaços menores entre as árvores adultas do dossel, cujas copas não chegam a se tocar) e dossel fechado (DF, onde há uma sobreposição ou um entrelaçamento das copas das árvores do dossel e do sub-bosque) (veja a Figura 1). A interpretação respeitou uma avaliação preliminar, abrangendo várias situações possíveis fora das áreas de amostragem e permitindo criar um padrão visual de reconhecimento adequado à estrutura da floresta na região. Além disso, todas as amostras foram avaliadas por uma mesma pessoa, para evitar erros ou diferenças decorrentes de critérios pessoais. 
Clareira

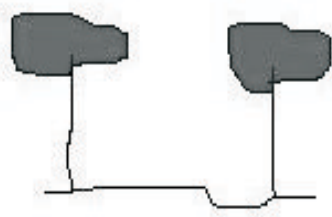

Dossel em regeneração

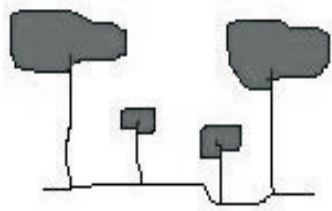

Dossel aberto

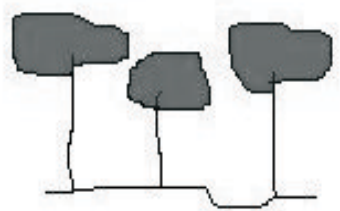

Dossel Fechado

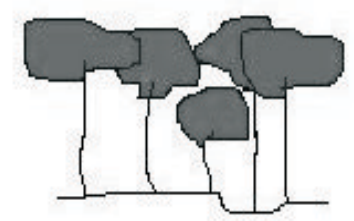

Figura 1 - Representação esquemática das quatro classes descritas na metodologia de ecounidades.

Figure 1 -Schematic representation of the four classes described in the eco-unit methodology.

As leituras da cobertura do dossel foram feitas com um densiômetro esférico convexo (D) de Lemmon (LEMMON, 1954), a norte, sul, leste e oeste, a $1 \mathrm{~m}$ do solo, em cada ponto de cada um dos locais, sempre por uma mesma pessoa. O densiômetro é composto por um espelho convexo, com o seu centro dividido em 24 quadrantes. Para a leitura, segundo Lemmon (1954), cada quadrante foi dividido mentalmente em quatro, e foram sistematicamente contados quantos quartos do quadrante refletiam o dossel; o total dos quadrantes foi somado e multiplicado por 1,04, derivando a estimativa de cobertura diretamente em porcentagem. Assim, foi obtida a cobertura de dossel, no local, pela soma da cobertura nos quatro pontos cardeais.

A medida de luminosidade na faixa visível do espectro foi feita com um luxímetro (L) analógico (marca Gossen, modelo Panlux Eletronic, com receptor de $4 \mathrm{~cm} \mathrm{de}$ diâmetro). Foram realizadas quatro leituras a $1 \mathrm{~m}$ do solo, em cada ponto de coleta: a norte, sul, leste e oeste. A média aritmética dos valores obtidos em cada ponto foi dividida pela média de valores obtidos em leituras a céu aberto, realizadas imediatamente antes e depois (com um intervalo menor que $5 \mathrm{~min}$ ) da entrada na floresta. Com esses valores foi obtida a proporção da luminosidade a céu aberto que penetra no sub-bosque.

Ainda para a análise do dossel, foram utilizados dois sistemas fotográficos. No primeiro (F32) foi utilizada uma câmera digital Sony Cyber-shot (DSC-S40) com lente de $32 \mathrm{~mm}$, fazendo duas fotografias de 1 megapixel, em nível, com a parte superior da câmera na direção norte, a $1 \mathrm{~m}$ do solo (com o auxílio de tripé). As fotos de cada ponto, com melhor contraste entre o dossel e o céu, foram submetidas ao Programa Adobe Photoshop 7.0, seguindo-se a metodologia de Engelbrecht e Herz (2001), com adaptações: (1) mudança da imagem para escala de cinza, na configuração do modo da imagem; (2) regulagem do contraste para +100 (sempre) e o brilho para - 100 (quando possível, tentando regulálo o mais próximo da foto original, utilizando-se a opção "visualização prévia"); (3) correção, quando necessário, de pequenos reflexos em galhos, troncos e folhas que assumiram a coloração branca e que seriam considerados como parte do dossel aberto, utilizando a ferramenta de "laço" e preenchendo o interior com preto; e (4) obter a informação de cobertura de dossel através do histograma da imagem, que foi dividida em duas tonalidades de cor (branco e preto), em que a porcentagem do preto equivale à cobertura de dossel.

No segundo sistema (F8), foi utilizada uma câmera digital Nikon Coolpix 8700 com lente de $8 \mathrm{~mm}$ (Fish eye, Nikon FC-E9), que fornece uma abertura de $180^{\circ}$, fazendo fotografias de 2 megapixels, em nível, com a parte superior da câmera na direção norte, a $1 \mathrm{~m}$ do solo (com o auxílio de tripé). As fotos de cada ponto com melhor contraste entre o dossel e o céu foram submetidas ao programa Gap Light Analyzer 2.0 (FRAZER et al., 1999), de acordo com o seguinte protocolo: (1) editar as configurações do programa conforme o local do experimento, (2) registrar a imagem, fixando um mesmo padrão para todas as fotos, (3) escolher o azul como plano de cor de fundo, (4) regular o limiar da imagem para 200, (5) fazer a correção, quando necessário, de pequenos reflexos em galhos, troncos e folhas que assumiram a coloração branca e que seriam considerados como parte do dossel aberto, (6) realizar os cálculos para cobertura de copa e de luminosidade que atravessa o dossel e (7) obter a porcentagem de cobertura de dossel e a porcentagem de luz total que atravessa a cobertura, medido em mols $/ \mathrm{m} 2 / \mathrm{d}$.

Os dados obtidos para comparar as diferentes metodologias foram submetidos a um teste de Wilcoxon $(\mathrm{p}<0,05)$ para medir a cobertura de dossel feita pelo $\mathrm{D}$, F8 e F32 e a um teste de correlação de Spearman ( $<<0,05)$, para medir quanto de luz penetra no sub-bosque utilizando

R. Árvore, Viçosa-MG, v.32, n.2, p.377-385, 2008 
o L e F8. Para comparar a estrutura do dossel do reflorestamento com o da floresta primária, em cada uma das metodologias utilizadas foi realizado um Teste- $t$ $(\mathrm{p}<0,05)$. Todas as análises estatísticas foram feitas pelo programa STATISTICA 6.0.

\section{RESULTADOS E DISCUSSÃO}

Os dados qualitativos das ecounidades foram comparados graficamente com as metodologias que

M1

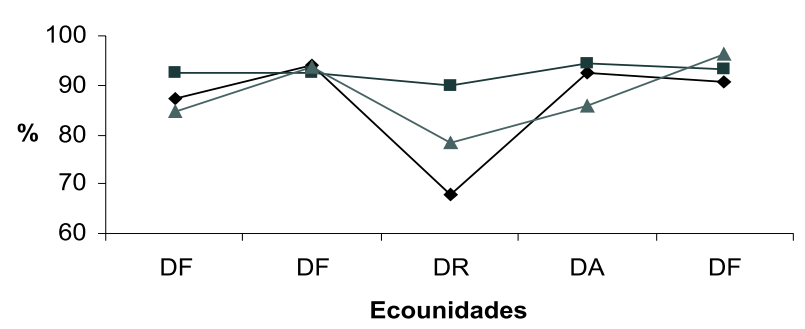

M2

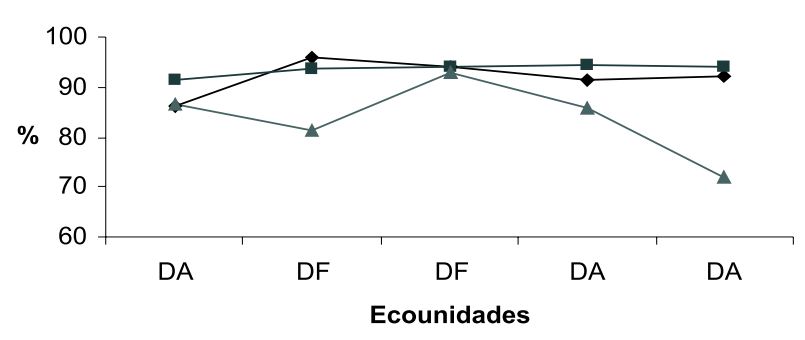

M3

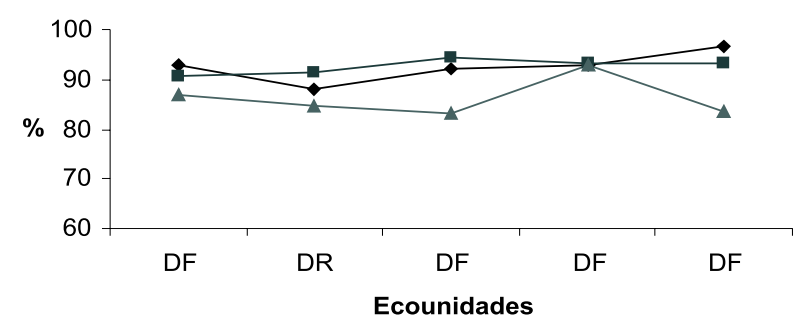

mostram a cobertura de dossel: D, F8 e F32 (Figura 2) e com as metodologias que medem a quantidade de luz que penetra no sub-bosque: L e F8 (Figura 3). A classificação em quatro categorias foi muito ampla, não permitindo detectar padrões nas ecounidades em relação aos outros métodos. Maior divisão de subclasses e amostragens com intervalo espacial menor poderia permitir a comparação dos métodos.

R1

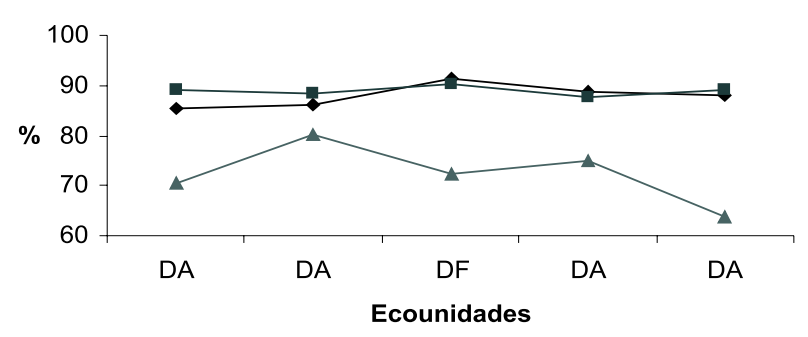

R2

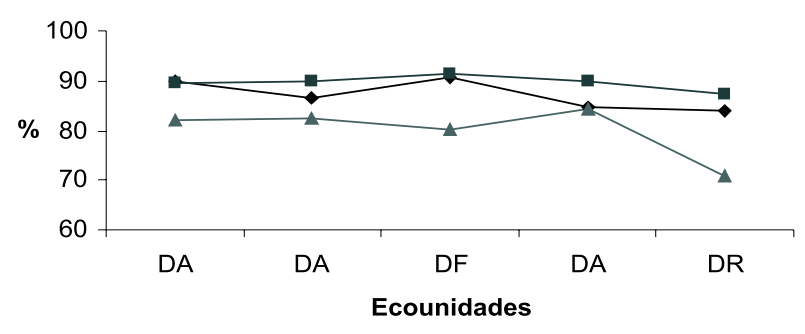

R3

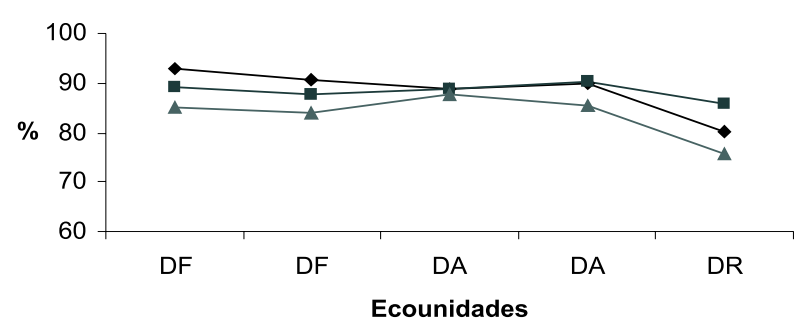

Figura 2 - Comparação entre as ecounidades $(C L=$ clareira, $\mathrm{DR}=$ dossel em regeneração, $\mathrm{DA}=$ dossel aberto e $\mathrm{DF}=$ dossel fechado) e as porcentagens de cobertura de dossel ( $\mathrm{D}=$ densiômetro, F8 = foto com lente de $8 \mathrm{~mm}$ e F32 = foto com lente de $32 \mathrm{~mm}$ ) em cada transecto. M são os transectos da floresta madura e R, os transectos do reflorestamento, no Parque Estadual Mata dos Godoy, PR.

Figure 2 - Comparison between the eco-units $(C L=$ clearing, $D R=$ regeneration gap, $D A=$ open gap and $D F=$ closed gap $)$ and canopy cover percentage $(D=$ densiometer, $F 8=$ picture with lens $8 \mathrm{~mm}$ and $F 32=$ picture with lens $32 \mathrm{~mm})$ in each transect. $M$ represents the mature forest transects and $R$ represents the reforestation transects at the Mata dos Godoy State Park, PR.

R. Árvore, Viçosa-MG, v.32, n.2, p.377-385, 2008 
M1

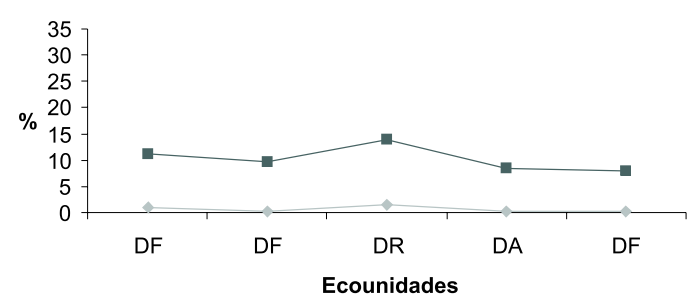

M2

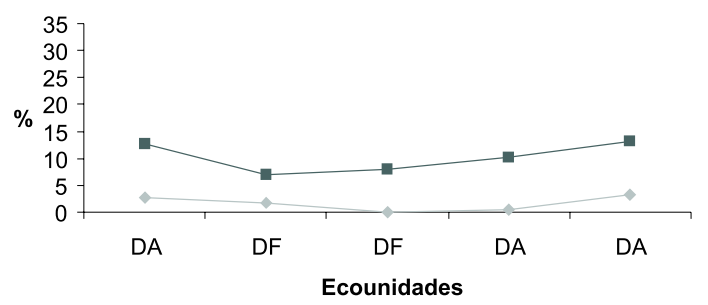

M3

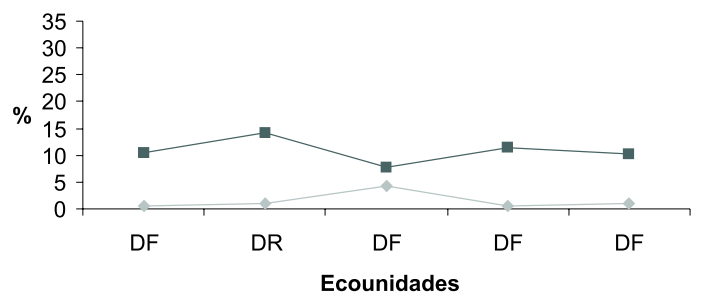

R1

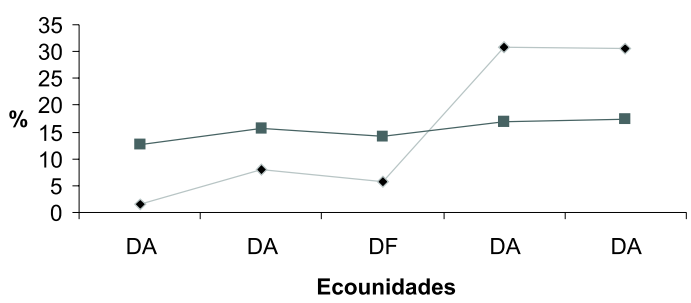

R2

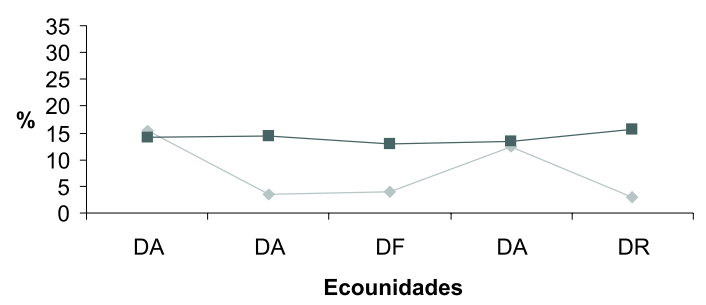

R3

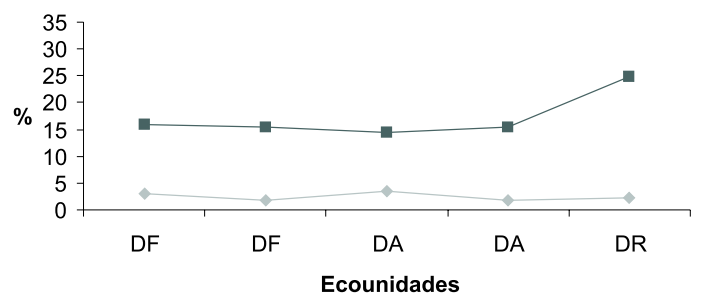

Figura 3 - Comparação entre as ecounidades $(C L=$ clareira, $D R=$ dossel em regeneração, $D A=$ dossel aberto e $D F=$ dossel fechado) e as porcentagens da luminosidade que penetra no sub-bosque ( $\mathrm{L}=$ luxímetro e $\mathrm{F} 8=$ foto com lente de $8 \mathrm{~mm}$ ) em cada transecto, em que M são os transectos da floresta madura e R, os transectos do reflorestamento, no Parque Estadual Mata dos Godoy, PR.

Figure 3 - Comparison between the eco-units $(C L=$ clearing, $D R=$ regeneration gap, $D A=$ open gap and $D F=$ closed gap $)$ and the percentages of luminosity that penetrate the understore $(L=$ light meter and $F 8=$ picture with lens 8 $\mathrm{mm})$ in each transect. $M$ represents the mature forest transects and $R$ represents the reforestation transects, at the Mata dos GodoyState Park, PR.

As análises dos dados de cobertura de dossel, no Local-M, no Local-R e quando agrupados os dados dos dois locais, não exibiram diferenças entre D e F8. Contudo, as duas metodologias foram diferentes da F32 (com p < <0,05). As metodologias F8 e D apresentaram amplas imagens esféricas, diferentemente de F32, que só conseguiu registrar pequena área plana. Os resultados indicam que F32 é mais sensível a pequenas variações que ocorrem no dossel, o que pode ser observado nas grandes variações das suas médias (Figura 4). Dados semelhantes foram relatados por Engelbrecht e Herz
(2001). Esses autores encontraram maior erro para calcular valores de luz que penetram no sub-bosque utilizando uma câmera com lente de grande angular (lente de 32 $\mathrm{mm}$ ) do que uma com lente olho de peixe (lente de $8 \mathrm{~mm}$ ).

O teste de correlação entre L e F8, na intensidade de luz que penetra no sub-bosque, não apresentou, em nenhum dos dois locais, uma correlação significativa (Figura 5ab); o mesmo é válido quando os dados dos dois locais foram agrupados (Figura 5c). Possivelmente, a sensibilidade do luxímetro a pequenos fachos localizados 
de luz que penetram o dossel pode ser responsável pelas grandes variações nas suas médias, mesmo sendo realizadas quatro medidas em cada ponto, de maneira similar à lente F32.
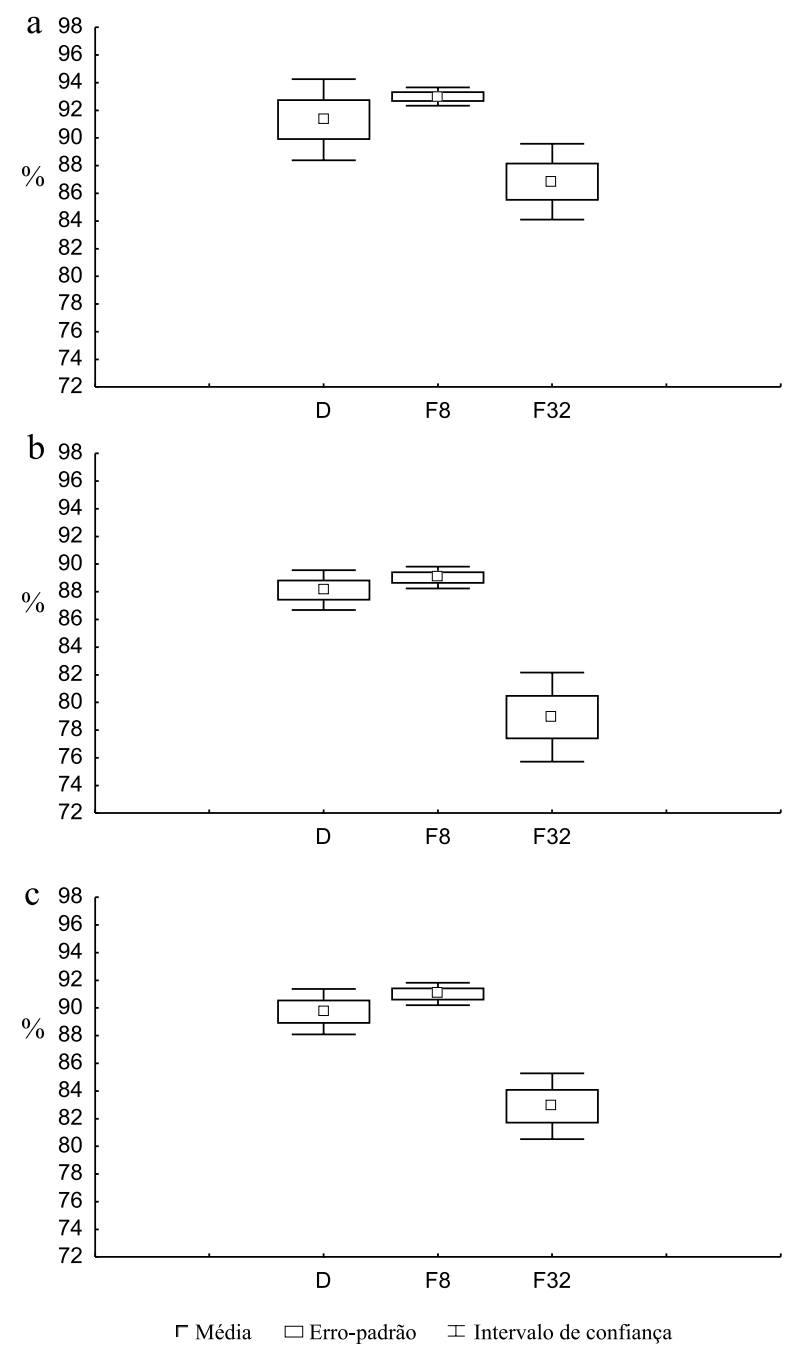

Figura 4 - Comparação de três metodologias, apresentando média, erro-padrão e desvio-padrão nos locais estudados: (a) na floresta madura, (b) no reflorestamento e (c) nos dois locais juntos. D $=$ densiômetro, $\mathrm{F} 8$ = fotografia com lente de 8 $\mathrm{mm}$ e F32 = fotografia com lente de $32 \mathrm{~mm}$, no Parque Estadual Mata dos Godoy, PR.

Figure 4 - Comparison of three methods, presenting average, standard error and standard deviation in the studied sites (a) mature forest, $(b)$ reforestation area and (c) both sites together. $D=$ densiometer, $F 8=$ picture with $8 \mathrm{~mm}$ lens and F32= picture with $32 \mathrm{~mm}$ lens, at the Mata dos Godoy State Park, PR.

R. Árvore, Viçosa-MG, v.32, n.2, p.377-385, 2008
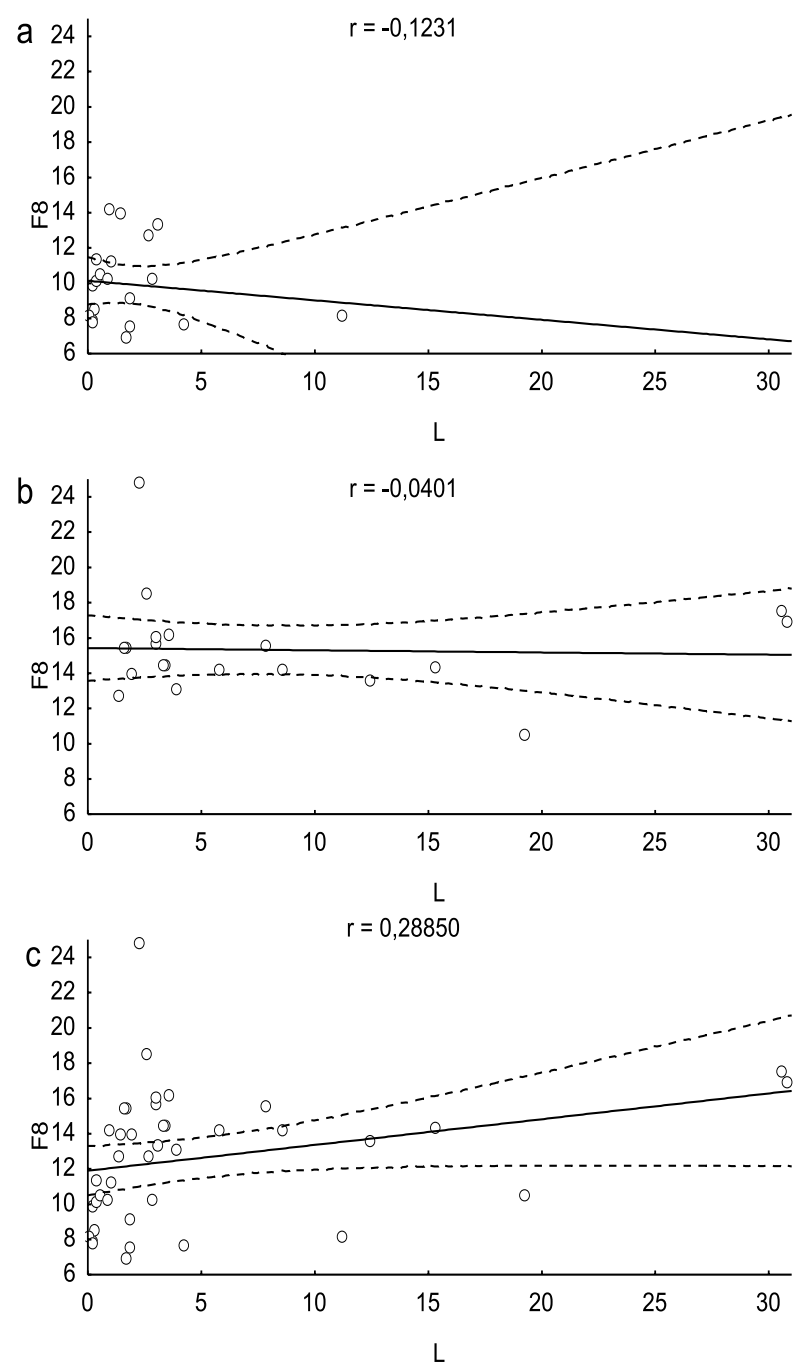

Figura 5 - Correlações $(\mathrm{r}=$ beta da reta) entre $\mathrm{F} 8=$ foto com lente de $8 \mathrm{~mm}$ e $\mathrm{L}=$ luxímetro, com $95 \%$ de grau de confiança: (a) na floresta madura, (b) no reflorestamento e (c) nos dois locais juntos, no Parque Estadual Mata dos Godoy, PR.

Figure 5 -Correlations ( $r=$ beta of the line) between $F 8=$ photo with $8 \mathrm{~mm}$ lens and L= light meter, with $95 \%$ of reliable degree in the (a) mature forest, (b) reforestation and (c) both sites, at the Mata dos Godoy State Prak, PR.

Comparando os locais a partir das EUs, foi maior a porcentagem de DF no Local-M (65\%) e de DA no Local-R (70\%), e a porcentagem de DR (10\%) e CL (0\%) foi igual nos dois locais, mostrando, assim, maior cobertura de dossel na mata. Com as demais metodologias, sempre foi encontrada diferença entre os locais, seguindo-se o mesmo padrão das EUs de 
maior cobertura na mata do que no reflorestamento (Figura 6ab), demonstrando que todos os métodos foram eficientes para detectar essa diferença entre o dossel da floresta madura e o do reflorestamento. Dados de Harper (1989) descrevem um padrão semelhante ao encontrado, em que florestas mais jovens (Local-R) teriam folhas menos adensadas nas copas das árvores, proporcionando menor cobertura de dossel do que em florestas mais antigas (Local-M). Isso pode ser explicado também pelo adensamento dos indivíduos, pois o maior espaçamento entre os indivíduos no reflorestamento proporcionaria um ambiente mais seco, que aumentaria a transpiração das plantas, fazendo que estas percam mais as folhas (BIANCHINI et al., 2001).

As metodologias foram tabeladas quanto à sua praticidade, custo, obtenção de dados em campo e seus tratamentos, como descrito no Anexo 1, evidenciando-se, assim, as vantagens e desvantagens de cada uma, podendo ser escolhida a metodologia de acordo com as necessidades e condições do trabalho a ser realizado.
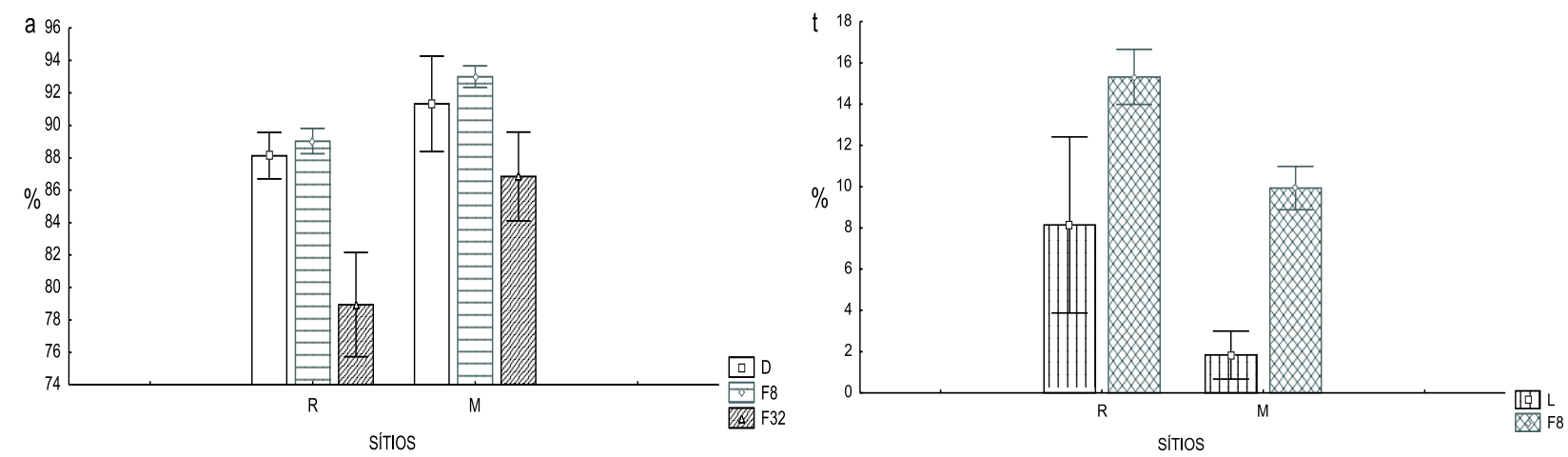

Figura 6 - Comparação entre cada metodologia e os locais estudados no Parque Estadual Mata dos Godoy, PR, em que M = floresta madura e $\mathrm{R}=$ reflorestamento. (a) $\mathrm{D}=$ densiômetro, $\mathrm{F} 8=$ foto com lente de $8 \mathrm{~mm}$ e $\mathrm{F} 32=$ foto com lente de $32 \mathrm{~mm}$ e (b) $\mathrm{L}=$ luxímetro e F8. As barras representam o desvio-padrão de $95 \%$.

Figure 6 -Comparison between each methodology and the studied sites at the Mata dos Godoy State Park-PR,, where $M=$ mature forest and the $R=$ reforestation. (a) $D=$ densiometer, $F 8=$ photo with $8 \mathrm{~mm}$ lens and $F 32=$ photo with $32 \mathrm{~mm}$ lens and $(b) L=$ light meter and $F 8$. The bars represent the standard deviation of $95 \%$.

\section{CONCLUSÃO}

Comparando as metodologias, pode-se comprovar que não houve diferença entre o uso de densiômetro e a fotografia com lentes de $8 \mathrm{~mm}$ em nenhum dos dois ambientes, apesar de a F8 demonstrar menor variação das médias, ou seja, resultados mais apurados. Já a fotografia com lentes de $32 \mathrm{~mm}$ apresentou grandes variações, ou seja, alta sensibilidade a pequenas variações na formação do dossel, o que é uma conseqüência da pequena área que F32 consegue registrar para ser analisada.

O mesmo se aplica à captação de luz no subbosque, tendo a fotografia com lentes de $8 \mathrm{~mm}$ e o luxímetro apresentado dados diferentes, em que F8 demonstrou menor variação nas suas médias e L teve grande variação, indicando, assim, menor precisão que a F8, causada, principalmente, por sua pequena área de captação de luz.
Todas as metodologias detectaram diferenças entre o dossel da floresta madura e o do reflorestamento, mostrando uma boa eficiência em conseguir distinguir esses dois ambientes, seguindo sempre um mesmo padrão, que apresenta maior cobertura de dossel na mata do que no reflorestamento.

As metodologias foram tabeladas quanto à sua praticidade, custo, obtenção de dados em campo e seus tratamentos, como descrito na Tabela 1, mostrando, assim, as vantagens e desvantagens de cada uma; pode ser escolhida a metodologia, de acordo com as necessidades e condições do trabalho a ser realizado.

\section{AGRADECIMENTOS}

À equipe do LABRE, pela ajuda em campo, especialmente a Edson M. Francisco e Odair C. Pavão; e ao Instituto Ambiental do Paraná, por permitir o acesso ao Parque Estadual Mata dos Godoy.

R. Árvore, Viçosa-MG, v.32, n.2, p.377-385, 2008 


\section{REFERÊNCIAS}

ALVARENGA, A. A. et al. Effects of different light levels on the initial growth and photosynthesis of Croton urucurana Baill. In southeastern Brazil. Revista Árvore, v.27, n.1, p.53-57, 2003.

BIANCHINI, E. et al. Spatial and temporal variation in a Tropical Semi-deciduous Forest. Brazilian Archives of Biology and Technology, v.44, p.269-276, 2001.

CAMPBELL, C. S.; NORMAN, J. M. The description and measurement of plant canopy structure. In: RUSSELL, G.; MARSHALL, B.; JARVIS, P. G. Plant canopies: Their growth, form and function. Cambridge: Cambridge University, 1989. 178p.

DENSLOW, J. S. et al. Growth responses of tropical shrubs to treefall gap environments. Ecology, v.71, n.1, p.525-532, 1990.

ENGELBRECHT, B. M. J.; HERZ, H. M. Evaluation of different methods to estimate understorey light conditions in tropical forests. Journal of Tropical Ecology, v.17, p.207-224, 2001.

FRAZER, G. W. et al. Gap Light Analyzer (GLA), Version 2.0. Simon Fraser University, Burnaby, British Columbia, and the Institute of Ecossistem Studies, Millbrook, New York, 1999. 36p.

HARPER, J. L. Canopies as populations. In: RUSSELL, G.; MARSHALL, B.; JARVIS, P. G. Plant canopies: Their growth, form and function. Cambridge: Cambridge University, 1989. 178p.
LAMB, D. et al. Rejoining habitat remnants: Restoring degraded rainforest lands. In: LAURENCE, W. F.; BIERREGAARD, R. O. Tropical forest remmants. Chicago: The University of Chicago, 1997. p.366-385.

LEMMON, P. E. A new instrument for measuring forest overstory density. Jounal of Forestry, v.55, n.9, p.667-668, 1957.

LIMA JR., E. C. et al. Aspectos fisioanatômicos de plantas jovens de Cupania vernalis Camb. submetidas a diferentes níveis de sombreamento. Revista Árvore, v.30, n.1, p.33-41, 2006.

RINCON, E.; HUANTE, P. Growth responses of tropical deciduous tree seedlings to contrasting light conditions. Trees: Structure and Function, v.7, p.202-207, 1993.

SILVEIRA, M. A vegetação do Parque Estadual Mata dos Godoy. In: TOREZAN, J. M. D. (Org.) Ecologia do Parque Estadual Mata dos Godoy. Londrina: ITEDES, 2006. p.19-27.

TURTON, S. M.; FREIBURGER, H. J. Edge and aspect effects on the microclimate of a small tropical forest remnant on the Atherton Tableland, northeastern Australia. In: LAURENCE, W. F.; BIERREGAARD, R. O. Tropical forest remmants. Chicago: The University of Chicago, 1997. p.45-54.

VICENTE, R. F. O Parque Estadual Mata dos Godoy. In: TOREZAN, J. M. D. (Org.) Ecologia do Parque Estadual Mata dos Godoy. Londrina: ITEDES, 2006. p.13-18. 
ANEXO 1

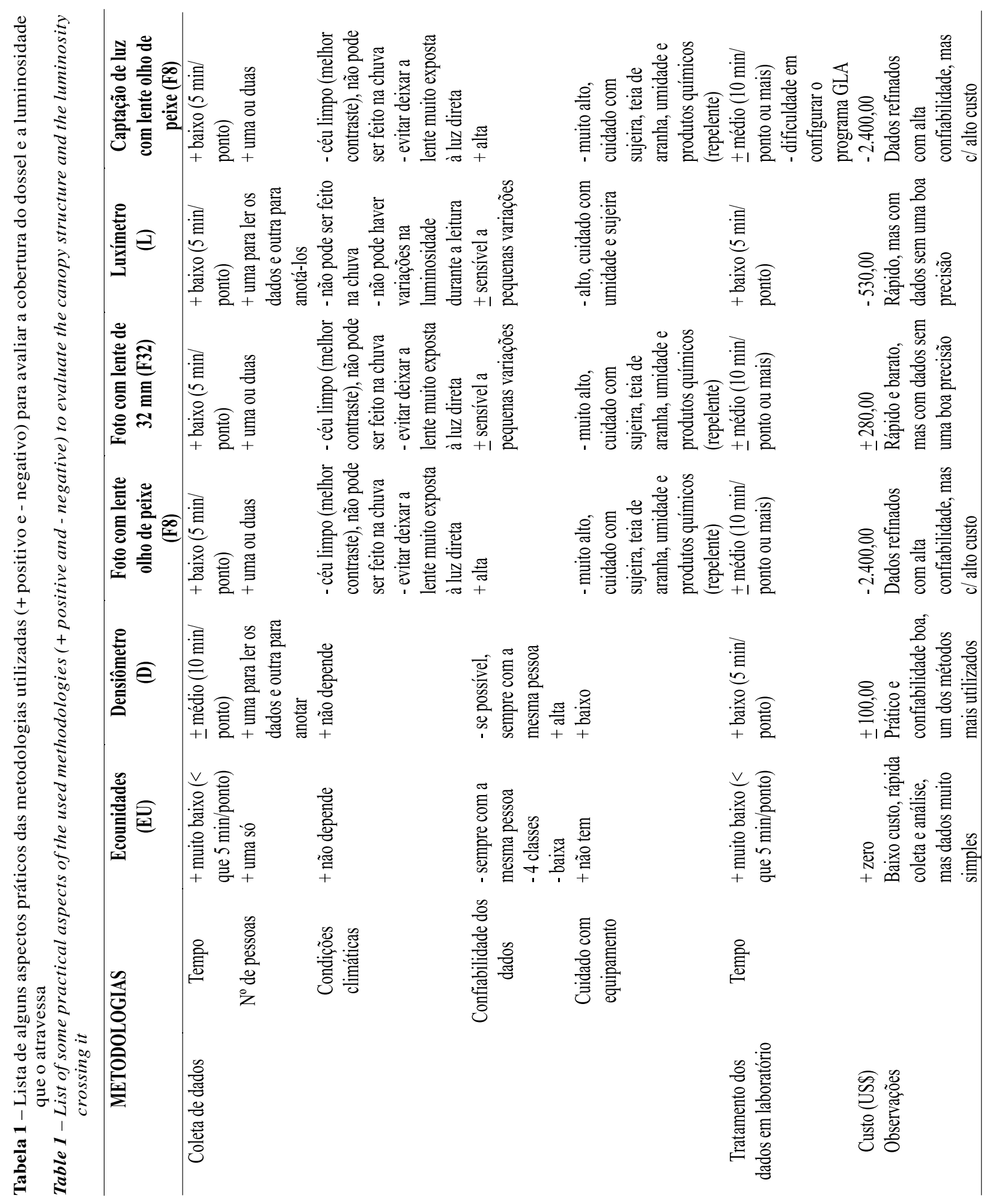

R. Árvore, Viçosa-MG, v.32, n.2, p.377-385, 2008 